\title{
T-duality constraints on higher derivatives revisited
}

\author{
Olaf Hohm ${ }^{a, b}$ and Barton Zwiebach ${ }^{a}$ \\ ${ }^{a}$ Center for Theoretical Physics, Massachusetts Institute of Technology, \\ Cambridge, MA, 02139 U.S.A. \\ ${ }^{b}$ Simons Center for Geometry and Physics, Stony Brook University, \\ Stony Brook, NY, 11794-3636 U.S.A. \\ E-mail: ohohm@mit.edu, zwiebach@mit.edu
}

\begin{abstract}
We ask to what extent are the higher-derivative corrections of string theory constrained by T-duality. The seminal early work by Meissner tests T-duality by reduction to one dimension using a distinguished choice of field variables in which the bosonic string action takes a Gauss-Bonnet-type form. By analyzing all field redefinitions that may or may not be duality covariant and may or may not be gauge covariant we extend the procedure to test T-duality starting from an action expressed in arbitrary field variables. We illustrate the method by showing that it determines uniquely the first-order $\alpha^{\prime}$ corrections of the bosonic string, up to terms that vanish in one dimension. We also use the method to glean information about the $\mathcal{O}\left(\alpha^{\prime 2}\right)$ corrections in the double field theory with Green-Schwarz deformation.
\end{abstract}

KeYwords: Global Symmetries, String Duality

ArXiv EPRINT: 1510.00005 


\section{Contents}

1 Introduction $\quad 1$

2 Cosmological reduction and field redefinitions 4

2.1 Reduction including lapse function and general field redefinitions 4

2.2 Field redefinitions, lapse gauge fixing and canonical form for the action $\quad 7$

$\begin{array}{lll}2.3 O(d, d) \text { covariant field redefinitions } & 12\end{array}$

$\begin{array}{lll}3 & \text { Bosonic string theory at } \mathcal{O}\left(\alpha^{\prime}\right) \text { revisited } & 15\end{array}$

4 Green-Schwarz term and T-duality at $\mathcal{O}\left(\alpha^{2}\right) \quad 19$

5 Conclusions $\quad 24$

\section{Introduction}

The T-duality symmetries of string theory have implications for the low-energy effective field theories obtained after compactification on a torus. The full bosonic string theory compactified on a $d$-dimensional torus has a discrete $O(d, d ; \mathbb{Z})$ duality group [1]. On the other hand, the low-energy effective field theory for the massless degrees of freedom has an enhanced $O(d, d ; \mathbb{R})$ continuous global symmetry. This symmetry was long-recognized in the two-derivative approximation to the effective field theory [2-5], where the global symmetry transformations take a simple form [6].

More nontrivially, using string field theory and the symmetries of S-matrix elements of massless states it was shown in $[4,5]$ that the continuous $O(d, d ; \mathbb{R})$ global symmetry survives $\alpha^{\prime}$ corrections to the effective field theory. These arguments were recently reviewed and elaborated to show that the $O(d, d ; \mathbb{R})$ symmetry in the low-energy effective field theory of heterotic strings is also preserved to all orders in $\alpha^{\prime}[7]$. Thus the continuous duality symmetry is valid for the effective field theory of the full classical string theory. The arguments prove the existence of the duality symmetry but do not yield the associated field transformations. When the action is written in generally coordinate invariant form these dualities acquire $\alpha^{\prime}$ corrections, are rather complicated and are not well understood.

The manifest display of global duality symmetries in the presence of $\alpha^{\prime}$ corrections is a natural subject of study in double field theory formulations [8-12] of the low-energy limits of string theories. Motivated by the recent progress in encoding $\alpha^{\prime}$ corrections in double field theory [13-16] we revisit here some aspects of the continuous T-duality symmetry of effective field theories.

Given an effective field theory for a metric field, a $b$-field, and a dilaton, one wants to know if this theory has a duality symmetry, by which we mean an $O(d, d, \mathbb{R})$ symmetry 
arising upon dimensional reduction on a torus $T^{d}$. If the theory includes higher derivative corrections and is written in generally coordinate invariant notation, the answer is not easily found. A test of T-duality to first order in $\alpha^{\prime}$ was performed by Meissner [17] using the generally-covariant effective field theory of bosonic strings and performing a (cosmological) reduction to one dimension. In the analysis of [17] it seems necessary to bring this action into a particular Gauss-Bonnet-type form by covariant field redefinitions before reduction. After reduction one aims to rewrite the action in terms of a generalized metric and a duality invariant dilaton. This final step requires further field redefinitions that cannot originate from covariant redefinitions before reduction. The test [17] is a necessary condition for T-duality but does not prove it. An obvious limitation of this method is that certain linear combinations of terms that are nonzero in arbitrary dimensions sometimes become zero upon reduction to one dimension. These combinations may fail to be T-duality covariant, but no constraint arises from the reduction.

A similar analysis of the T-duality constraints on $\alpha^{\prime}$ corrections was recently given by Godazgar and Godazgar [18], who consider the reduction on an arbitrary $d$-dimensional torus but truncate to the scalar degrees of freedom, hence giving a necessary but not sufficient condition for T-duality. As in the analysis of Meissner, realizing an $O(d, d, \mathbb{R})$ symmetry seems to lead to a preferred field basis in the original gravity action. It would be useful to have full control of the field redefinition freedom. (See [19] for related investigations of T-duality and $\alpha^{\prime}$ corrections.)

It is the purpose of this paper to extend the discussion of Meissner to make it fully systematic and to deal in all generality with field redefinitions. Indeed, while the analysis of [17] begins with a 'minimal' form of the $\mathcal{O}\left(\alpha^{\prime}\right)$ effective action, the method requires the use of covariant field redefinitions to recast the action in a form where dimensional reduction and integration by parts yields terms with no more than first-order time derivatives. This takes a fair amount of work, and the resulting action is significantly more complicated than the original, minimal one. Since the existence of T-duality symmetry is independent of field redefinitions, a complete method should work with the simplest starting point. Here we will develop such a method. Moreover, we also show that field redefinitions allow for previously unnoticed simplifications of the duality covariant forms of the reduced action.

In the remainder of this introduction we outline our method and results. The dimensional reduction for the metric and $b$-field is based on an ansatz of the form

$$
g_{\mu \nu}=\left(\begin{array}{cc}
-n^{2}(t) & 0 \\
0 & g_{i j}(t)
\end{array}\right), \quad b_{\mu \nu}=\left(\begin{array}{cc}
0 & 0 \\
0 & b_{i j}(t)
\end{array}\right) .
$$

Here $n(t)$ is the 'lapse' function. One can use the diffeomorphism symmetry to set $n(t)=1$ but one has to remember the field equation for $n(t)$. This field equation is needed to perform field redefinitions on the reduced action. After that freedom is taken into account one may set $n(t)=1$, at which point the action becomes a function of matrices $L, M$, defined in terms of the matrices $g_{i j}$ and $b_{i j}$ :

$$
L \equiv g^{-1} \dot{g}, \quad M \equiv g^{-1} \dot{b} .
$$


The action will also depend on time derivatives of $L$ and $M$, as well as on the dualityinvariant dilaton $\Phi$ and its time derivatives.

In the next step of the procedure one uses the metric and $b$-field equations of motion to eliminate via field redefinitions any appearance of $\dot{L}$ and $\dot{M}$ terms from the action. Terms with higher time derivatives of $L$ or $M$, if any, would require integration by parts, until one is able to use the equations of motion. We describe identities that allow one to remove terms with first derivatives or powers of first derivatives of the dilaton. Terms with two time derivatives on the dilaton can be eliminated using the dilaton equation to motion and, using integration by parts, so can terms with more than two time derivatives on the dilaton. The end result is a simplified reduced action that is just a function of traces of powers of $L$ and $M$.

This result must be set equal to the most general duality invariant one-dimensional action plus terms that correspond to the 'lapse' field redefinition. Up to equations of motion we demonstrate that the duality covariant action can be written in terms of traces of powers of first time derivatives of the generalized metric. Any term involving time derivatives of the dilaton can be redefined away. This quickly implies a very simple result: the number of parameters in the $2 k$-derivative part of the duality covariant one-dimensional action $(k \geq 2)$ is equal to the number $p(k)$ of partitions of $k$. The most general lapse field redefinition includes an additional set of parameters. The test shows T-duality is possible if one can adjust all of those parameters to obtain equality with the simplified reduced action.

We illustrate our method with two examples. In the first we reconsider the $\mathcal{O}\left(\alpha^{\prime}\right)$ corrections of bosonic string theory. Up to field redefinitions these corrections are determined by eight coefficients. We test T-duality and show that the correct T-duality covariant action emerges uniquely up to a two-fold ambiguity: there are two linear combinations of terms that are not constrained because their reduction to one dimension gives zero. ${ }^{1}$ The final $O(d, d)$ covariant four-derivative action at order $\mathcal{O}\left(\alpha^{\prime}\right)$ can be brought to the form

$$
S^{(1)}=\frac{1}{16} \int d t e^{-\Phi}\left(\operatorname{tr} \dot{\mathcal{S}}^{4}-\frac{1}{2}\left(\operatorname{tr} \dot{\mathcal{S}}^{2}\right)^{2}\right), \quad \mathcal{S} \equiv \eta \mathcal{H}
$$

where $\mathcal{H}$ is the generalized metric taking values in $O(d, d)$ and $\eta$ is the $O(d, d)$ invariant metric. This action is equivalent to that given by Meissner in [17] up to duality covariant field redefinitions that eliminate all terms with dilaton time derivatives.

In the second example we consider the 'doubled $\alpha^{\prime}$ geometry' of [13]. It has nontrivial $\alpha^{\prime}$ corrections and an exact duality symmetry that does not have $\alpha^{\prime}$ corrections. In this theory it is the general coordinate transformations that receive $\alpha^{\prime}$ corrections. Rewritten in terms of a conventional metric and $b$-field, however, the duality symmetries will have $\alpha^{\prime}$ corrections. Information gathered recently [21] indicates that the $\mathcal{O}\left(\alpha^{\prime}\right)$ corrections cubic in fields are those of a Chern-Simons form based on a torsionless gravitational connection. We ask here if the simplest form of the action consistent with this information is T-duality covariant to all orders. We use our method to show that this minimal action fails to be

\footnotetext{
${ }^{1}$ For reductions to dimensions $D>1$ these combinations are not zero, and so almost surely those linear combinations are inconsistent with T-duality. If that is the case, the $\mathcal{O}\left(\alpha^{\prime}\right)$ action of the bosonic string is fully determined by continuous duality.
} 
T-duality covariant to $\mathcal{O}\left(\alpha^{\prime 2}\right)$. This demonstrates that the double field theory of [13] must contain additional corrections.

\section{Cosmological reduction and field redefinitions}

In this section we develop a method to test if a given action is consistent with T-duality. The first step is to perform the dimensional reduction to one dimension (section 2.1) and to bring that action into canonical form (section 2.2). We discuss lapse redefinitions while working in the gauge where the lapse function is set equal to one. We show that, up to general field redefinitions, the action can be written in terms of $L$ and $M$, see (1.2). In particular, there are no time derivatives of $L$ or $M$, nor dilaton time derivatives. The second step is to match the reduced action to a one-dimensional duality covariant action, whose terms we classify up to duality-covariant field redefinitions (section 2.3). The matching condition is stated in equation (2.45).

\subsection{Reduction including lapse function and general field redefinitions}

We begin by performing the (cosmological) reduction to one dimension of the standard two-derivative, low-energy action for the bosonic string:

$$
S=\int d^{D} x \sqrt{-g} e^{-2 \phi}\left(R+4(\partial \phi)^{2}-\frac{1}{12} H^{2}\right) .
$$

Here $H_{\mu \nu \rho}=3 \partial_{[\mu} b_{\nu \rho]}$ is the field strength for the $b$-field. In the reduction we drop the dependence on all internal coordinates, leaving only the dependence on time $t$,

$$
x^{\mu}=\left(t, x^{i}\right), \quad \partial_{i}=0 .
$$

For the metric, antisymmetric tensor and (scalar) dilaton we have

$$
g_{\mu \nu}=\left(\begin{array}{cc}
-n^{2}(t) & 0 \\
0 & g_{i j}(t)
\end{array}\right), \quad b_{\mu \nu}=\left(\begin{array}{cc}
0 & 0 \\
0 & b_{i j}(t)
\end{array}\right), \quad \phi=\phi(t) .
$$

Before proceeding with the computation of the reduction, it is useful to examine the residual diffeomorphisms of the reduction ansatz. Since we have kept the lapse function $n(t)$, we still have time reparametrization invariance. This diffeomorphism symmetry $t \rightarrow$ $t-\lambda(t)$ acts as

$$
\begin{aligned}
\delta_{\lambda} n & =\partial_{t}(\lambda n), \\
\delta_{\lambda} g_{i j} & =\lambda \dot{g}_{i j}, \\
\delta_{\lambda} b_{i j} & =\lambda \dot{b}_{i j}, \\
\delta_{\lambda} \phi & =\lambda \dot{\phi},
\end{aligned}
$$

where we use dots or $\partial_{t}$ to denote time derivatives. Note that all fields except for $n(t)$ transform as scalars under time reparameterizations. The field $n(t)$ transforms as a density. For any field $A$ that transforms as a scalar,

$$
\delta_{\lambda} A=\lambda \partial_{t} A,
$$


one can readily verify that the combination $n^{-1} \partial_{t}$ is a covariant time derivative and thus $n^{-1} \partial_{t} A$ is also a scalar:

$$
\delta_{\lambda}\left(n^{-1} \partial_{t} A\right)=\lambda \partial_{t}\left(n^{-1} \partial_{t} A\right) .
$$

It is also quickly seen that for any such scalar $A$, the combination $n A$ is a scalar density:

$$
\delta_{\lambda}(n A)=\partial_{t}(\lambda n A)
$$

It will we useful for us to define a different dilaton by

$$
e^{-\Phi} \equiv \sqrt{\operatorname{det}\left(g_{i j}\right)} e^{-2 \phi} .
$$

Since $g_{i j}$ and $\phi$ transform as scalars, both $e^{-\Phi}$ and $\Phi$ are scalars:

$$
\delta_{\lambda} \Phi=\lambda \dot{\Phi} .
$$

Let us now begin the calculation of the reduction. If we reduce (2.1) to one dimension, using the definition of the dilaton $\Phi$,

$$
S=\int d t n e^{-\Phi}\left(R+4(\partial \phi)^{2}-\frac{1}{12} H^{2}\right) .
$$

In order to compute the various terms in the action, we need the Christoffel symbols, whose non-vanishing components are

$$
\Gamma_{i j}^{0}=\frac{1}{2 n^{2}} \dot{g}_{i j}, \quad \Gamma_{i 0}^{j}=\frac{1}{2} g^{j k} \dot{g}_{i k}, \quad \Gamma_{00}^{0}=\frac{\dot{n}}{n} .
$$

For the lower-index version $\Gamma_{\alpha \mu \nu} \equiv g_{\alpha \beta} \Gamma_{\mu \nu}^{\beta}$ we get

$$
\Gamma_{0 i j}=-\Gamma_{i j 0}=-\frac{1}{2} \dot{g}_{i j}, \quad \Gamma_{000}=-n \dot{n} .
$$

The non-vanishing components of the Riemann tensor are then found to be

$$
\begin{aligned}
R_{i j k l} & =\frac{1}{2 n^{2}} \dot{g}_{k[i} \dot{g}_{j] l} \\
R_{0 i 0 j} & =-\frac{1}{2} n \partial_{t}\left(\frac{1}{n} \dot{g}_{i j}\right)+\frac{1}{4} g^{k l} \dot{g}_{k i} \dot{g}_{l j} .
\end{aligned}
$$

After some calculation using the above, the scalar curvature $R$ is determined to be

$$
R=\frac{1}{n} \partial_{t}\left(g^{i j} \frac{1}{n} \partial_{t} g_{i j}\right)+\frac{1}{4}\left(\frac{1}{n} g^{i j} \dot{g}_{i j}\right)^{2}+\frac{1}{4} g^{i j} g^{k l} \frac{1}{n} \dot{g}_{i l} \frac{1}{n} \dot{g}_{k j} .
$$

It is manifest that each term here is a scalar.

It is convenient to define, using matrix notation,

$$
L \equiv g^{-1} \dot{g}, \quad M \equiv g^{-1} \dot{b},
$$


so that $L^{i}{ }_{j}=g^{i k} \dot{g}_{k j}$ and $M^{i}{ }_{j}=g^{i k} \dot{b}_{k j}$. All our matrices have the row index up and the column index down. Note that

$$
\mathcal{L} \equiv n^{-1} L, \quad \text { and } \quad \mathcal{M} \equiv n^{-1} M
$$

transform as scalars. In the gauge $n=1, \mathcal{L}$ becomes $L$ and $\mathcal{M}$ becomes $M$. Note also the simple identities

$$
g^{-1} \ddot{g}=\dot{L}+L^{2}, \quad g^{-1} \ddot{b}=\dot{M}+L M .
$$

Using the above result for the scalar curvature, noting that the dilaton relation (2.8) gives

$$
\dot{\phi}=\frac{1}{2}\left(\dot{\Phi}+\frac{1}{2} \operatorname{tr} L\right)
$$

and reducing the kinetic term for the antisymmetric tensor,

$$
-\frac{1}{12} H_{\mu \nu \rho}^{2}=-\frac{1}{n^{2}} \frac{1}{4} \operatorname{tr} M^{2}
$$

we find that the two-derivative action in (2.10) becomes

$$
S=\int d t \frac{1}{n} e^{-\Phi}\left(-\dot{\Phi}^{2}+\frac{1}{4} \operatorname{tr}\left(L^{2}-M^{2}\right)\right) .
$$

The reparameterization invariance is manifest because the action equals

$$
S=\int d \operatorname{tn} e^{-\Phi}\left(-\left(\frac{1}{n} \dot{\Phi}\right)^{2}+\frac{1}{4} \operatorname{tr}\left(\mathcal{L}^{2}-\mathcal{M}^{2}\right)\right),
$$

which is written in terms of the covariant time derivatives of dilaton, metric and $b$-field, multiplied by the density $n$. We find that the metric and $b$-field equations of motion take the form

$$
\begin{aligned}
\dot{\mathcal{L}} & =\partial_{t}\left(n^{-1} L\right)=n^{-1}\left(M^{2}+\dot{\Phi} L\right), \\
\dot{\mathcal{M}} & =\partial_{t}\left(n^{-1} M\right)=n^{-1}(M L+\dot{\Phi} M),
\end{aligned}
$$

while the dilaton equation of motion is

$$
\frac{d}{d t}\left(n^{-1} \dot{\Phi}\right)=\frac{1}{2} n^{-1}\left(\dot{\Phi}^{2}+\frac{1}{4} \operatorname{tr}\left(L^{2}-M^{2}\right)\right) .
$$

The equation of motion for the lapse $n$ is quite simple: it sets the Lagrangian density equal to zero, which means

$$
-\dot{\Phi}^{2}+\frac{1}{4} \operatorname{tr}\left(L^{2}-M^{2}\right)=0 .
$$

We can finally bring the action into manifestly $O(d, d)$ covariant form. We first recall that $\eta \mathcal{H}$, where $\eta$ is the invariant metric and $\mathcal{H}$ the generalized metric, takes the form

$$
\eta \mathcal{H}=\left(\begin{array}{cc}
b g^{-1} & g-b g^{-1} b \\
g^{-1} & -g^{-1} b
\end{array}\right) \text {. }
$$


From this one may verify by a quick calculation that

$$
\operatorname{tr}(\eta \dot{\mathcal{H}})^{2}=2 \operatorname{tr}\left(M^{2}-L^{2}\right) .
$$

Comparing with the dimensionally reduced action (2.20), one finds that the latter can be written as

$$
S=\int d t \frac{1}{n} e^{-\Phi}\left(-\dot{\Phi}^{2}-\frac{1}{8} \operatorname{tr}(\eta \dot{\mathcal{H}})^{2}\right),
$$

which is now manifestly $O(d, d)$ invariant. Both $\Phi$ and $n(t)$ are inert under $O(d, d)$ transformations.

\subsection{Field redefinitions, lapse gauge fixing and canonical form for the action}

In order to test if a generally covariant action has T-duality symmetry we reduce to one dimension. In this reduction we keep the lapse function $n(t)$ as a variable. Since T-duality in two-derivative actions is understood, the purpose here is to deal with the generally covariant higher derivative couplings that appear in the effective field theory as terms in a power series in $\alpha^{\prime}$.

Having reduced the full action the next step is to simplify it in a canonical way by using field redefinitions. The field redefinitions will be viewed as perturbative in $\alpha^{\prime}$. Our rule will be to use metric, $b$-field and dilaton equations of motion in order to eliminate all terms with two or more derivatives of these fields. In order to implement these field redefinitions we can simply view them as allowed substitutions in the higher-derivative terms. From (2.22) and (2.23) we have the substitutions:

$$
\begin{aligned}
n^{-1} \dot{\mathcal{L}} & \rightarrow n^{-2}\left(M^{2}+\dot{\Phi} L\right), \\
n^{-1} \dot{\mathcal{M}} & \rightarrow n^{-2}(M L+\dot{\Phi} M), \\
n^{-1} \frac{d}{d t}\left(n^{-1} \dot{\Phi}\right) & \rightarrow \frac{1}{2} n^{-2}\left(\dot{\Phi}^{2}+\frac{1}{4} \operatorname{tr}\left(L^{2}-M^{2}\right)\right) .
\end{aligned}
$$

All objects to the left and right of the arrow are scalars. There is one more substitution possible, based on the lapse field equation (2.24),

$$
\dot{\Phi}^{2} \rightarrow \frac{1}{4} \operatorname{tr}\left(L^{2}-M^{2}\right) .
$$

After we use all these substitutions we try to see if the resulting action has T-duality. At this point we can use the gauge $n(t)=1$, and thus the test of T-duality amounts to trying to write the resulting action in terms of $\mathcal{H}$, which encapsulates $g_{i j}(t)$ and $b_{i j}(t)$, and the dilaton $\Phi$.

In practice we can simplify a bit our work by letting $n=1$ before doing the reduction to one dimension and before using the field equations for the metric, $b$-field and dilaton. After setting $n=1$ the replacements corresponding to these field equations become

$$
\begin{aligned}
\dot{L} & \rightarrow M^{2}+\dot{\Phi} L \\
\dot{M} & \rightarrow M L+\dot{\Phi} M, \\
\ddot{\Phi} & \rightarrow \frac{1}{2}\left(\dot{\Phi}^{2}+\frac{1}{4} \operatorname{tr}\left(L^{2}-M^{2}\right)\right) \rightarrow \frac{1}{4} \operatorname{tr}\left(L^{2}-M^{2}\right),
\end{aligned}
$$


where we used the lapse equation in the last replacement. Since in this way we lose the $n$ field equation, we must recall that we have the ability to do the lapse-related field redefinition (2.29). Setting $n=1$ before reduction and use of the equations of motion gives the same result as setting $n=1$ after reduction and use of equations of motion. This is because all terms in the action are time-reparameterization scalars and for them all appearances of $n$ are in the form $n^{-1} \partial_{t}$. Setting $n=1$ then just leaves the time derivatives that would have been obtained otherwise.

Let us discuss more explicitly the replacement rule. If we vary the metric in the two-derivative Lagrangian $\mathcal{L}^{(2)}\left(S=\int e^{-\Phi} \mathcal{L}\right)$ we find an expression of the form

$$
\delta \mathcal{L}^{(2)}=\operatorname{tr}\left(-\frac{1}{2} g^{-1} \delta g\left(\dot{L}-\left(M^{2}+\dot{\Phi} L\right)\right)\right) .
$$

Now assume that in the full higher-derivative Lagrangian we find a term of the form

$$
\mathcal{L}=\mathcal{L}^{(2)}+\alpha^{\prime} \operatorname{tr}(X \dot{L})+\ldots,
$$

where the dots represent other terms of order $\alpha^{\prime}$ or higher. A trivial rewriting expresses this as the sum of two terms:

$$
\left.\mathcal{L}=\mathcal{L}^{(2)}+\alpha^{\prime} \operatorname{tr}\left(X\left(\dot{L}-\left(M^{2}+\dot{\Phi} L\right)\right)\right)+\alpha^{\prime} \operatorname{tr}\left(X\left(M^{2}+\dot{\Phi} L\right)\right)\right)+\ldots
$$

It now follows from (2.31) that if we redefine the metric with

$$
-\frac{1}{2} g^{-1} \delta g=-\alpha^{\prime} X
$$

the underlined term above is cancelled, and we are left with a new Lagrangian $\mathcal{L}^{\prime}$ given by

$$
\left.\mathcal{L}^{\prime}=\mathcal{L}^{(2)}+\alpha^{\prime} \operatorname{tr}\left(X\left(M^{2}+\dot{\Phi} L\right)\right)\right)+\ldots
$$

Comparing with (2.32) we see that the effect of the field redefinition was to implement the replacement $\dot{L} \rightarrow M^{2}+\dot{\Phi} L$, as claimed. Note also that, in principle, the metric redefinition should be performed everywhere, not only in the two-derivative action. Nevertheless, since the redefinition is itself of order $\alpha^{\prime}$, the effect of the redefinition on the $\mathcal{O}\left(\alpha^{\prime}\right)$ terms will be order $\alpha^{\prime 2}$ terms. The analysis in section 3 is only to order $\alpha^{\prime}$, so there is no need to consider such terms. In section 4 the analysis is of order $\alpha^{\prime 2}$, and we will carefully consider the field redefinitions that include both a part proportional to $\alpha^{\prime}$ and a part proportional to $\alpha^{\prime 2}$. This will be facilitated by starting with a duality covariant Lagrangian (4.16) that does not have $\mathcal{O}\left(\alpha^{\prime}\right)$ terms.

Having removed terms with two derivatives on the fields another important set of identities allows us to remove terms with powers of $\dot{\Phi}$. Assume $X=X(L, M)$ is a function of $L$ and $M$ only and is of degree $k: X(\lambda L, \lambda M)=\lambda^{k_{X}} X(L, M)$. We can then manipulate a term of the form $\dot{\Phi} X$ in the Lagrangian as follows:

$$
\int d t e^{-\Phi} \dot{\Phi} X=-\int d t \partial_{t}\left(e^{-\Phi}\right) X=\int d t e^{-\Phi} \dot{X}
$$


Given that $X$ is of degree $k_{X}$, if we take the time derivative and use the substitutions in (2.30) to eliminate the $\dot{L}$ and $\dot{M}$ terms we will find

$$
\dot{X}=k_{X} \dot{\Phi} X+X^{\prime},
$$

where prime denotes the action of taking a time derivative and letting $\dot{L} \rightarrow M^{2}$ and $\dot{M} \rightarrow M L$. As a result we have

$$
\int d t e^{-\Phi} \dot{\Phi} X=\int d t e^{-\Phi}\left(k_{X} \dot{\Phi} X+X^{\prime}\right),
$$

which means that as terms in the Lagrangian we eliminate the $\dot{\Phi} X$ term via the equivalence

$$
\dot{\Phi} X \simeq \frac{1}{\left(1-k_{X}\right)} X^{\prime} .
$$

Here and in the following we denote by $\simeq$ equalities that hold up to equations of motion and integrations by parts. The above identity fails for $k_{X}=1$, but this case will not be relevant, as it would correspond to a term with two derivatives and we are interested in higher-derivative terms. As examples of the use of this identity consider two forms of $X$, both of degree three:

$$
\begin{aligned}
\dot{\Phi} \operatorname{tr}\left(L^{3}\right) & \simeq-\frac{3}{2} \operatorname{tr}\left(M^{2} L^{2}\right), \\
\dot{\Phi} \operatorname{tr}\left(M^{2} L\right) & \simeq-\frac{1}{2} \operatorname{tr}\left(M L M L+M^{2} L^{2}+M^{4}\right) .
\end{aligned}
$$

By a similar analysis, this time using the dilaton replacement (last line in (2.30)), one can show that

$$
\dot{\Phi}^{2} X \simeq-\frac{1}{2 k_{X}-1}\left(\frac{1}{4} \operatorname{tr}\left(L^{2}-M^{2}\right) X-\frac{2}{k_{X}} X^{\prime \prime}\right)
$$

Here $X^{\prime \prime}=\left(X^{\prime}\right)^{\prime}$, using the definition of prime given above. Since $k_{X}=0$ is not of interest and $k_{X}$ is an integer, the above formula always gives a well defined equivalence. As examples we record

$$
\begin{aligned}
\dot{\Phi}^{2} \operatorname{tr}\left(L^{2}\right) & \simeq-\frac{1}{12} \operatorname{tr}\left(L^{2}-M^{2}\right) \operatorname{tr}\left(L^{2}\right)+\frac{2}{3} \operatorname{tr}\left(M L M L+M^{2} L^{2}+M^{4}\right), \\
\dot{\Phi}^{2} \operatorname{tr}\left(L^{2}-M^{2}\right) & \simeq-\frac{1}{12}\left(\operatorname{tr}\left(L^{2}-M^{2}\right)\right)^{2} .
\end{aligned}
$$

For arbitrary powers of $\dot{\Phi}$ we can use the following relation, derived by exactly the same methods:

$$
\frac{1}{2}\left(3-p-2 k_{X}\right) \dot{\Phi}^{p} X \simeq \frac{p-1}{8} \operatorname{tr}\left(L^{2}-M^{2}\right) \dot{\Phi}^{p-2} X+\dot{\Phi}^{p-1} X^{\prime} .
$$

This relates a term with $\dot{\Phi}^{p}$ to terms with $\dot{\Phi}^{p-1}$ and $\dot{\Phi}^{p-2}$ and can be used recursively. Note that (2.39) follows from for $p=1$, and (2.41) follows for $p=2$, after using the $p=1$ result. This equation shows that we can always eliminate the $\dot{\Phi}$ dependence of terms using 
field equations. The prefactor on the left-hand side indicates that for $p=1, k_{X}=1$ or for $p=3, k_{X}=0$ the relation fails to help eliminate $\dot{\Phi}^{p} X$. But those cases correspond to terms with two and three derivatives, respectively, and are of no interest to us. The upshot of this analysis is that, by the use of field redefinitions, the dimensionally reduced action can be written as a function of $L$ and $M$ with no extra time derivatives and without any dilaton time derivatives.

As we mentioned while introducing (2.29), we simplify the reduced action using the lapse equation of motion to replace, for arbitrary $Y$,

$$
\dot{\Phi}^{2} Y \simeq \frac{1}{4} \operatorname{tr}\left(L^{2}-M^{2}\right) Y .
$$

We could use (2.41) instead, but this is more complicated and not needed at this stage. In order to explain this point, let us consider the general ansatz that needs to be solvable in order for duality invariance to be possible. Schematically, the matching equation reads

$($ reduction to $D=1) \simeq($ general 1D duality-invariant action $)+\left(\dot{\Phi}^{2}-\frac{1}{4} \operatorname{tr}\left(L^{2}-M^{2}\right)\right) X$,

where the last term accounts for lapse redefinitions and $X$ is an arbitrary function of $L, M$, and $\dot{\Phi}$. This form makes it manifest that the use of (2.44) is legal in the simplification of the left-hand side of the matching equation. Indeed, a term $\dot{\Phi}^{2} Y$ on the left-hand side can be trivially rewritten as

$$
\dot{\Phi}^{2} Y=\frac{1}{4} \operatorname{tr}\left(L^{2}-M^{2}\right) Y+\left(\dot{\Phi}^{2}-\frac{1}{4} \operatorname{tr}\left(L^{2}-M^{2}\right)\right) Y
$$

and we can ignore the second term as long as $X$ is general. If the reduction to $1 \mathrm{D}$ with all its simplifications has been carried out, and given that the general 1D duality-invariant action has no dilaton time derivatives (section 2.3), the only dilaton time derivatives in (2.45) are in the second term of the right-hand side. At this point the equivalence (2.41) and, more generally, (2.43), both valid up to field redefinitions, is needed to eliminate such dependence. This is why we use the symbol $\simeq$ in $(2.45)$.

To illustrate the simplification procedure for the reduction of the action to $D=1$, consider the reduction of Riemann-squared. Using the non-vanishing components of the Riemann tensor one quickly finds

$$
R_{\mu \nu \rho \sigma}^{2}=R^{i j k l} R_{i j k l}+4\left(g^{00}\right)^{2} R_{0}{ }^{j}{ }_{0 i} R_{0}{ }^{i}{ }_{0 j} .
$$

It follows immediately from the first equation in (2.13) that

$$
R^{i j k l} R_{i j k l}=\frac{1}{8}\left(\operatorname{tr} \mathcal{L}^{2}\right)^{2}-\frac{1}{8} \operatorname{tr} \mathcal{L}^{4}
$$

Moreover, a few lines of calculation using the second equation in (2.13) shows that

$$
R_{0}{ }^{i}{ }_{0 j}=-\frac{1}{2} n^{2}\left(n^{-1} \dot{\mathcal{L}}+\frac{1}{2} \mathcal{L}^{2}\right)^{i}{ }_{j}
$$


All in all Riemann-squared gives

$$
\begin{aligned}
R_{\mu \nu \rho \sigma}^{2} & =\frac{1}{8}\left(\operatorname{tr} \mathcal{L}^{2}\right)^{2}-\frac{1}{8} \operatorname{tr} \mathcal{L}^{4}+\operatorname{tr}\left(n^{-1} \dot{\mathcal{L}}+\frac{1}{2} \mathcal{L}^{2}\right)^{2} \\
& =\operatorname{tr}\left(\left(n^{-1} \dot{\mathcal{L}}\right)^{2}+n^{-1} \dot{\mathcal{L}} \mathcal{L}^{2}+\frac{1}{8} \mathcal{L}^{4}\right)+\frac{1}{8}\left(\operatorname{tr} \mathcal{L}^{2}\right)^{2}
\end{aligned}
$$

This is manifestly a scalar and, as expected, the time derivatives always appear accompanied by a factor of $n^{-1}$. Using the equation of motion for $g_{i j}$ and setting $n=1$ afterwards gives manifestly the same result as setting $n=1$ first and then using the simpler equations of motion. Setting first $n=1$ we have

$$
R_{\mu \nu \rho \sigma}^{2}=\operatorname{tr}\left(\dot{L}^{2}+\dot{L} L^{2}+\frac{1}{8} L^{4}\right)+\frac{1}{8}\left(\operatorname{tr} L^{2}\right)^{2} .
$$

Using now the replacement associated to the equation of motion (2.30) we get

$$
\begin{aligned}
R_{\mu \nu \rho \sigma} R^{\mu \nu \rho \sigma} \simeq & \operatorname{tr}\left(\frac{1}{8} L^{4}+M^{2} L^{2}+M^{4}\right)+\frac{1}{8}\left(\operatorname{tr}\left(L^{2}\right)\right)^{2} \\
& +\dot{\Phi} \operatorname{tr}\left(L^{3}+2 M^{2} L\right)+\dot{\Phi}^{2} \operatorname{tr}\left(L^{2}\right) .
\end{aligned}
$$

Employing next (2.40) and the lapse equation replacement we get

$$
\begin{aligned}
R_{\mu \nu \rho \sigma} R^{\mu \nu \rho \sigma} \simeq & \operatorname{tr}\left(\frac{1}{8} L^{4}+M^{2} L^{2}+M^{4}\right)+\frac{1}{8}\left(\operatorname{tr}\left(L^{2}\right)\right)^{2} \\
& +\operatorname{tr}\left(-M L M L-\frac{5}{2} M^{2} L^{2}-M^{4}\right)+\frac{1}{4} \operatorname{tr}\left(L^{2}-M^{2}\right) \operatorname{tr}\left(L^{2}\right)
\end{aligned}
$$

Simplifying, we finally get:

$$
R_{\mu \nu \rho \sigma} R^{\mu \nu \rho \sigma} \simeq \operatorname{tr}\left(\frac{1}{8} L^{4}-M L M L-\frac{3}{2} M^{2} L^{2}\right)+\frac{3}{8}\left(\operatorname{tr} L^{2}\right)^{2}-\frac{1}{4} \operatorname{tr} M^{2} \operatorname{tr} L^{2},
$$

where we recall that $\simeq$ means that the left-hand side and right-hand side are equal up to field redefinitions and integrations by parts. This expression will be needed in the later analysis.

Since we can do the reduction to one dimension without using the lapse function we collect a few formulae. The nonvanishing Christoffel symbols, $b$-field field strengths and curvatures are

$$
\begin{aligned}
\Gamma_{i j}^{0} & =\frac{1}{2} \dot{g}_{i j}, \quad \Gamma_{i 0}^{j}=\frac{1}{2} g^{j k} \dot{g}_{i k}, \quad \text { or } \quad \Gamma_{0 i j}=-\Gamma_{i j 0}=-\frac{1}{2} \dot{g}_{i j} . \\
H_{0}{ }^{i}{ }_{j} & =(M)^{i}{ }_{j}, \quad-\frac{1}{12} H_{\mu \nu \rho}^{2}=-\frac{1}{4} \operatorname{tr} M^{2} . \\
R_{i j k l} & =\frac{1}{2} \dot{g}_{k[i} \dot{g}_{j] l}, \quad R_{0}{ }^{i}{ }_{0 j}=-\frac{1}{2}\left(\dot{L}+\frac{1}{2} L^{2}\right)^{i}, \\
R_{00} & =-\frac{1}{2} \operatorname{tr}(\dot{L})-\frac{1}{4} \operatorname{tr}\left(L^{2}\right), \quad R^{i}{ }_{j}=\frac{1}{2}\left(\dot{L}+\frac{1}{2} L \operatorname{tr} L\right)^{i}{ }_{j}, \quad R=\operatorname{tr}(\dot{L})+\frac{1}{4}(\operatorname{tr} L)^{2}+\frac{1}{4} \operatorname{tr}\left(L^{2}\right) .
\end{aligned}
$$


To check the consistency of the reduction and our formulae we have examined in detail the covariant field equations for the metric, $b$-field, and dilaton. Their reduction give the equations of motion displayed above, with the lapse equation arising from the $g_{00}$ equation. We have also checked that we are not missing nontrivial field equations by setting $g_{0 i}=0$ and $b_{0 i}=0$ in the reduction ansatz.

\section{$2.3 O(d, d)$ covariant field redefinitions}

In this subsection we consider the duality covariant, one-dimensional, two-derivative action for $\mathcal{H}$ and $\Phi$ and then examine what are the possible duality covariant $\alpha^{\prime}$ corrections, up to field redefinitions. The action, setting $n=1$ in $(2.27)$, is

$$
S=\int d t e^{-\Phi}\left(-\dot{\Phi}^{2}-\frac{1}{8} \operatorname{tr} \dot{\mathcal{S}}^{2}\right), \quad \mathcal{S} \equiv \eta \mathcal{H}
$$

For brevity we have introduced $\mathcal{S}=\eta \mathcal{H}$, which satisfies $\mathcal{S}^{2}=1$. The equations of motion for $\mathcal{S}$ and $\Phi$ are then

$$
\begin{aligned}
\ddot{\mathcal{S}}+\mathcal{S}^{2}-\dot{\Phi} \dot{\mathcal{S}} & =0, \\
-2 \ddot{\Phi}+\dot{\Phi}^{2}-\frac{1}{8} \operatorname{tr} \dot{\mathcal{S}}^{2} & =0 .
\end{aligned}
$$

Thus, using $O(d, d)$ covariant field redefinitions we can always replace

$$
\begin{aligned}
\ddot{\mathcal{S}} & \rightarrow-\mathcal{S} \dot{\mathcal{S}}^{2}+\dot{\Phi} \dot{\mathcal{S}}, \\
\ddot{\Phi} & \rightarrow \frac{1}{2} \dot{\Phi}^{2}-\frac{1}{16} \operatorname{tr} \dot{\mathcal{S}}^{2} .
\end{aligned}
$$

We will next apply this freedom of duality covariant field redefinitions in the onedimensional $\mathcal{O}\left(\alpha^{\prime}\right)$ action found by Meissner. It is given by $\int d t e^{-\Phi} \mathcal{L}$ with Lagrangian [17]

$$
\mathcal{L}=\frac{1}{16} \operatorname{tr} \dot{\mathcal{S}}^{4}-\frac{1}{64}\left(\operatorname{tr} \dot{\mathcal{S}}^{2}\right)^{2}-\frac{1}{4} \dot{\Phi}^{2} \operatorname{tr} \dot{\mathcal{S}}^{2}-\frac{1}{3} \dot{\Phi}^{4}
$$

We will see that all dilaton terms can be removed by $O(d, d)$ invariant field redefinitions. We recall relation (2.43), which in $O(d, d)$ covariant language reads

$$
\frac{1}{2}\left(3-p-2 k_{X}\right) \dot{\Phi}^{p} X \simeq-\frac{p-1}{16} \operatorname{tr} \dot{\mathcal{S}}^{2} \dot{\Phi}^{p-2} X+\dot{\Phi}^{p-1} X^{\prime}
$$

For $X$ equal to a constant we get

$$
\dot{\Phi}^{k} \simeq \frac{1}{8}\left(\frac{k-1}{k-3}\right) \dot{\Phi}^{k-2} \operatorname{tr} \dot{\mathcal{S}}^{2}
$$

This allows us to trade the $\dot{\Phi}^{4}$ term in the above Lagrangian for a $\dot{\Phi}^{2} \operatorname{tr} \dot{\mathcal{S}}^{2}$ term. ${ }^{2}$ Note now that the second relation in (2.42) implies that

$$
\dot{\Phi}^{2} \operatorname{tr} \dot{\mathcal{S}}^{2} \simeq \frac{1}{24}\left(\operatorname{tr} \dot{\mathcal{S}}^{2}\right)^{2} .
$$

\footnotetext{
${ }^{2}$ Note, however, that we could not eliminate a $\dot{\Phi}^{3}$ term — but this is irrelevant because such term has three derivatives and is of no interest to us.
} 
Using these relations one quickly shows that the above Lagrangian (viewed as an addition to the two-derivative theory) is field-redefinition equivalent to the simpler

$$
\mathcal{L} \simeq \frac{1}{16} \operatorname{tr} \dot{\mathcal{S}}^{4}-\frac{1}{32}\left(\operatorname{tr} \dot{\mathcal{S}}^{2}\right)^{2}
$$

Classification of $\boldsymbol{O}(\boldsymbol{d}, \boldsymbol{d})$ invariants. We will classify all possible $O(d, d)$ invariant terms that can be added to the two-derivative action. These will be written in terms of $\mathcal{S}=\eta \mathcal{H}$, its derivatives, the dilaton $\Phi$ and its derivatives. Terms that differ by $O(d, d)$ covariant field redefinitions will be considered equivalent. The result is simple: terms are constructed by taking traces, or products of traces, of even powers of $\dot{\mathcal{S}}$. In particular, terms involving the dilaton time derivatives do not appear.

To begin note that $\mathcal{S}$ has zero trace (see (2.25)), and therefore so do all of its derivatives,

$$
\operatorname{tr}(\mathcal{S})=\operatorname{tr}(\dot{\mathcal{S}})=\operatorname{tr}(\ddot{\mathcal{S}})=0 .
$$

Moreover, since $\mathcal{S} \mathcal{S}=1$ we immediately learn that $\mathcal{S}$ and $\dot{\mathcal{S}}$ anticommute:

$$
\mathcal{S} \dot{\mathcal{S}}+\dot{\mathcal{S}} \mathcal{S}=0
$$

We first show that traces of odd powers of $\dot{\mathcal{S}}$ vanish. For this purpose we take a second derivative of the above equation to get

$$
2 \dot{\mathcal{S}} \dot{\mathcal{S}}+\ddot{\mathcal{S}} \mathcal{S}+\mathcal{S} \ddot{\mathcal{S}}=0 .
$$

Multiplying from the left by $(\dot{\mathcal{S}})^{2 k+1}$, with $k$ a non-negative integer, we find

$$
2(\dot{\mathcal{S}})^{2 k+3}+(\dot{\mathcal{S}})^{2 k+1} \ddot{\mathcal{S}} \mathcal{S}+(\dot{\mathcal{S}})^{2 k+1} \mathcal{S} \ddot{\mathcal{S}}=0
$$

Taking traces and using cyclicity, we have

$$
2 \operatorname{tr}\left(\dot{\mathcal{S}}^{2 k+3}\right)+\operatorname{tr}\left(\mathcal{S} \dot{\mathcal{S}}^{2 k+1} \ddot{\mathcal{S}}+\dot{\mathcal{S}}^{2 k+1} \mathcal{S} \ddot{\mathcal{S}}\right)=0 .
$$

Noting now with $(2.68)$ that $\mathcal{S} \dot{\mathcal{S}}^{2 k+1}=-\dot{\mathcal{S}}^{2 k+1} \mathcal{S}$, we learn that

$$
\operatorname{tr}\left(\dot{\mathcal{S}}^{2 k+3}\right)=0
$$

Since $\operatorname{tr}(\dot{\mathcal{S}})=0$, we have now proven, as we claimed, that

$$
\operatorname{tr}\left(\dot{\mathcal{S}}^{2 k+1}\right)=0, \quad \text { for } \quad k=0,1, \ldots
$$

There is no need to consider the use of $\ddot{\mathcal{S}}$ or higher time derivatives of $\mathcal{S}$. Using the $\mathcal{S}$ field equation we can implement the replacements in (2.61) to trade a double derivative of $\mathcal{S}$ for terms with $\mathcal{S}, \dot{\mathcal{S}}$ and $\dot{\Phi}$. We see that we must consider terms that also involve the undifferentiated $\mathcal{S}$. There is nothing that can be done with just $\mathcal{S}$, as it has zero trace, and once squared it equals the identity matrix. The question is if we can build some new duality invariant using $\mathcal{S}$ and $\dot{\mathcal{S}}$. The answer is no, as we show next. 
If we have a trace of a string of products of $\mathcal{S}$ 's and $\dot{\mathcal{S}}$ 's, using the anticommutativity of $\mathcal{S}$ and $\dot{\mathcal{S}}$ the term can be arranged so that all the $\mathcal{S}$ 's are near each other and thus, since $\mathcal{S}^{2}=1$, the only possible terms are of the form

$$
\operatorname{tr}\left(\mathcal{S} \dot{\mathcal{S}}^{k}\right)
$$

for $k$ a non-negative integer. It is straightforward to see that they vanish for all $k$, including $k=1$,

$$
\operatorname{tr}\left(\mathcal{S} \dot{\mathcal{S}}^{k}\right)=\operatorname{tr}\left(\mathcal{S} \dot{\mathcal{S}} \dot{\mathcal{S}}^{k-1}\right)=-\operatorname{tr}\left(\dot{\mathcal{S}} \mathcal{S} \dot{\mathcal{S}}^{k-1}\right)=-\operatorname{tr}\left(\mathcal{S} \dot{\mathcal{S}}^{k}\right)=0
$$

Here we used (2.68) in the second equality and cyclicity of the trace in the third equality.

The last issue we have to discuss is terms with time derivatives of the dilaton. Only first derivatives are relevant, since terms with two or more time derivatives of the dilaton can be reduced by using the dilaton field equation. But we have already seen that (2.63) allows us to get rid of such terms. Therefore there are no dilaton time derivatives in the one-dimensional duality invariant action, up to field redefinitions.

Our analysis implies that for four derivatives the most general duality covariant terms that, up to field redefinitions, can be added to the action are

$$
\mathcal{O}\left(\alpha^{\prime}\right): \quad a_{1} \operatorname{tr} \dot{\mathcal{S}}^{4}+a_{2}\left(\operatorname{tr} \dot{\mathcal{S}}^{2}\right)^{2}
$$

For six derivative terms we have

$$
\mathcal{O}\left(\left(\alpha^{\prime}\right)^{2}\right): \quad c_{1} \operatorname{tr} \dot{\mathcal{S}}^{6}+c_{2} \operatorname{tr} \dot{\mathcal{S}}^{4} \operatorname{tr} \dot{\mathcal{S}}^{2}+c_{3}\left(\operatorname{tr} \dot{\mathcal{S}}^{2}\right)^{3}
$$

The terms that arise at order $\left(\alpha^{\prime}\right)^{k-1}$ have $2 k$ derivatives and look like

$$
\mathcal{O}\left(\left(\alpha^{\prime}\right)^{k-1}\right): \quad c_{1} \operatorname{tr} \dot{\mathcal{S}}^{2 k}+c_{2} \operatorname{tr} \dot{\mathcal{S}}^{2 k-2} \operatorname{tr} \dot{\mathcal{S}}^{2}+c_{3} \operatorname{tr} \dot{\mathcal{S}}^{2 k-4}\left(\operatorname{tr} \dot{\mathcal{S}}^{2}\right)^{2}+c_{4} \operatorname{tr} \dot{\mathcal{S}}^{2 k-4} \operatorname{tr} \dot{\mathcal{S}}^{4}+\ldots
$$

Letting $z \equiv \dot{\mathcal{S}}^{2}$ and assuming that each factor in a product has its trace taken, the above expression is

$$
c_{1} z^{k}+c_{2} z^{k-1} z+c_{3} z^{k-2} z z+c_{4} z^{k-2} z^{2}+\ldots,
$$

making it clear that each summand can be associated with a partition of the integer $k$. Thus the number of independent coefficients in the $\mathcal{O}\left(\left(\alpha^{\prime}\right)^{k-1}\right)$ action is $p(k)$, the number of partitions of $k$. This is the number of coefficients in the part of the action with $2 k$ derivatives.

For reference we collect the first few independent invariants in terms of $L$ and $M$,

$$
\begin{aligned}
& \operatorname{tr}\left(\dot{\mathcal{S}}^{2}\right)= 2 \operatorname{tr}\left(-L^{2}+M^{2}\right) \\
& \operatorname{tr}\left(\dot{\mathcal{S}}^{4}\right)=2 \operatorname{tr}\left(L^{4}+2 M L M L-4 M^{2} L^{2}+M^{4}\right) \\
& \operatorname{tr}\left(\dot{\mathcal{S}}^{6}\right)=2 \operatorname{tr}\left(-L^{6}-6 M L^{3} M L+3 M L^{2} M L^{2}+6 M^{2} L^{4}\right. \\
&\left.\quad \quad-3 M^{2} L M^{2} L+6 M^{3} L M L-6 M^{4} L^{2}+M^{6}\right) .
\end{aligned}
$$




\section{Bosonic string theory at $\mathcal{O}\left(\alpha^{\prime}\right)$ revisited}

As an application and illustration of the general procedure developed above, we consider the bosonic string effective action including $\mathcal{O}\left(\alpha^{\prime}\right)$ corrections and investigate to what extent it is constrained by duality invariance in the reduction to one dimension. We start with the most general four-derivative action, up to field redefinitions, according to Tseytlin and Metsaev. This action contains eight terms and thus eight coefficients $\gamma_{i}, i=1, \ldots, 8$, and takes the form [20]:

$$
S(\gamma)=\int d^{D} x \sqrt{-g} e^{-2 \phi} \mathcal{L}(\gamma)
$$

where

$$
\begin{aligned}
\mathcal{L}(\gamma)= & \gamma_{1} R_{\mu \nu \rho \sigma}^{2}+\gamma_{2} H H R+\gamma_{3} H^{4}+\gamma_{4}\left(H_{\mu \nu}^{2}\right)^{2} \\
& +\gamma_{5}\left(H^{2}\right)^{2}+\gamma_{6} H_{\mu \nu}^{2} \partial^{\mu} \phi \partial^{\nu} \phi+\gamma_{7} H^{2}(\partial \phi)^{2}+\gamma_{8}(\partial \phi)^{4} .
\end{aligned}
$$

In here, the various terms are defined as follows:

$$
\begin{aligned}
H H R & \equiv H^{\mu \nu \lambda} H_{\lambda}^{\rho \sigma} R_{\mu \nu \rho \sigma} \\
H^{4} & \equiv H_{\mu \nu \rho}(H H H)^{\mu \nu \rho} \equiv H_{\mu \nu \rho} H_{\alpha}^{\mu}{ }^{\beta} H_{\beta}^{\nu}{ }^{\gamma} H_{\gamma}{ }^{\alpha} \\
H_{\mu \nu}^{2} & \equiv H_{\mu}{ }^{\alpha \beta} H_{\nu \alpha \beta} \\
\left(H_{\mu \nu}^{2}\right)^{2} & \equiv H_{\mu \nu}^{2} H^{2 \mu \nu} \\
H^{2} & \equiv H_{\mu \nu \rho} H^{\mu \nu \rho}
\end{aligned}
$$

Given the known result that the $\mathcal{O}\left(\alpha^{\prime}\right)$ action of bosonic string theory, up to field redefinitions, is [20]

$$
\int d^{D} x \sqrt{-g} e^{-2 \phi}\left(R_{\mu \nu \rho \sigma}^{2}-\frac{1}{2} H H R+\frac{1}{24} H^{4}-\frac{1}{8}\left(H_{\mu \nu}^{2}\right)^{2}\right)
$$

we know that

$$
\gamma_{1}=1, \quad \gamma_{2}=-\frac{1}{2}, \quad \gamma_{3}=\frac{1}{24}, \quad \gamma_{4}=-\frac{1}{8}, \quad \gamma_{5}=\gamma_{6}=\gamma_{7}=\gamma_{8}=0,
$$

is a duality invariant solution. We now want to see if this is the answer selected by the condition of duality invariance.

The strategy is to reduce the Lagrangian $\mathcal{L}(\gamma)$ down to one dimension using the field equations to eliminate $\dot{L}, \dot{M}, \ddot{\Phi}$ and $\dot{\Phi}^{2}$ terms. The result is equated to the general duality covariant terms plus the most general lapse redefinition, as explained in (2.45). The lapse redefinition takes the form

$$
\left(\dot{\Phi}^{2}-\frac{1}{4} \operatorname{tr}\left(L^{2}-M^{2}\right)\right)\left(b_{1} \operatorname{tr}\left(L^{2}\right)+b_{2} \operatorname{tr}\left(M^{2}\right)+b_{3}(\operatorname{tr} L)^{2}+b_{4} \dot{\Phi}^{2}+b_{5} \dot{\Phi}(\operatorname{tr} L)\right),
$$

where the terms in parenthesis are the most general terms we can write with two time derivatives, realizing that $\operatorname{tr}(M)=\operatorname{tr}(M L)=0$. The term with coefficient $b_{5}$ gives, up to 
equations of motion, precisely minus the contribution of the term with coefficient $b_{2}$. This is so because, up to equations of motion,

$$
e^{-\Phi}\left(\dot{\Phi}^{2}-\frac{1}{4} \operatorname{tr}\left(L^{2}-M^{2}\right)\right) \operatorname{tr}\left(M^{2}+\dot{\Phi} L\right) \simeq e^{-\Phi}\left(\dot{\Phi}^{2}-\frac{1}{4} \operatorname{tr}\left(L^{2}-M^{2}\right)\right) \operatorname{tr} \dot{L},
$$

and the term in the right hand side is a total total derivative, as can be checked integrating by parts the time derivative acting on $L$ and using the dilaton, metric, and $b$-field equations of motion. Therefore we can set $b_{5}$ equal to zero. Using (2.76) and (2.45) the dimensionally reduced Lagrangian should then be writable as

$$
\begin{aligned}
\left.\mathcal{L}(\gamma)\right|_{1 d} \simeq & a_{1} \operatorname{tr}(\eta \dot{\mathcal{H}})^{4}+a_{2}\left(\operatorname{tr}(\eta \dot{\mathcal{H}})^{2}\right)^{2} \\
& +\left(\dot{\Phi}^{2}-\frac{1}{4} \operatorname{tr}\left(L^{2}-M^{2}\right)\right)\left(b_{1} \operatorname{tr}\left(L^{2}\right)+b_{2} \operatorname{tr}\left(M^{2}\right)+b_{3}(\operatorname{tr} L)^{2}+b_{4} \dot{\Phi}^{2}\right),
\end{aligned}
$$

which will constrain the $\gamma$ coefficients. This is our key equation. The right-hand side can be evaluated with (2.80) and (2.41) to give

$$
\begin{aligned}
\operatorname{rhs} \simeq & \operatorname{tr}\left(2 a_{1} L^{4}+4 a_{1} M L M L-8 a_{1} M^{2} L^{2}+2 a_{1} M^{4}\right) \\
& +4 a_{2}\left(\operatorname{tr} L^{2}\right)^{2}+4 a_{2}\left(\operatorname{tr} M^{2}\right)^{2}-8 a_{2}\left(\operatorname{tr} M^{2}\right)\left(\operatorname{tr} L^{2}\right) \\
& +b_{1}\left(-\frac{1}{3} \operatorname{tr}\left(L^{2}-M^{2}\right) \operatorname{tr} L^{2}+\frac{2}{3} \operatorname{tr}\left(M L M L+M^{2} L^{2}+M^{4}\right)\right) \\
& +b_{2}\left(-\frac{1}{3} \operatorname{tr}\left(L^{2}-M^{2}\right) \operatorname{tr} M^{2}+\frac{2}{3} \operatorname{tr}\left(M L M L+M^{2} L^{2}+M^{4}\right)\right) \\
& +b_{3}\left(-\frac{1}{3} \operatorname{tr}\left(L^{2}-M^{2}\right)(\operatorname{tr} L)^{2}+\frac{2}{3}\left(\operatorname{tr} M^{2}\right)^{2}+\frac{4}{3} \operatorname{tr}\left(M^{2} L\right) \operatorname{tr} L\right) \\
& +b_{4} \frac{1}{12}\left(\operatorname{tr}\left(L^{2}-M^{2}\right)\right)^{2} .
\end{aligned}
$$

Now we must evaluate the left-hand side by computing the cosmological reduction of (3.2). The Riemann-squared term was given in (2.54). The HHR invariant yields

$$
\begin{aligned}
H H R & =\operatorname{tr}\left(M^{2} L^{2}+\frac{1}{2} M L M L+2 \dot{L} M^{2}\right) \\
& =\operatorname{tr}\left(M^{2} L^{2}+\frac{1}{2} M L M L+2 M^{4}\right)+2 \dot{\Phi} \operatorname{tr}\left(M^{2} L\right) \\
& \simeq \operatorname{tr}\left(-\frac{1}{2} M L M L+M^{4}\right) .
\end{aligned}
$$

For the $H^{4}$ invariants we find

$$
H^{4}=3 \operatorname{tr}\left(M^{4}\right), \quad\left(H_{\mu \nu}^{2}\right)^{2}=4 \operatorname{tr}\left(M^{4}\right)+\left(\operatorname{tr}\left(M^{2}\right)\right)^{2}, \quad\left(H^{2}\right)^{2}=9\left(\operatorname{tr} M^{2}\right)^{2} .
$$

We need to calculate the terms with dilaton derivatives. Here we can use the lapse equation (as well as the other equations) to replace

$$
\dot{\Phi}^{2} \rightarrow \frac{1}{4} \operatorname{tr}\left(L^{2}-M^{2}\right)
$$


and thus simplify some of the calculations. First, one finds

$$
\begin{aligned}
H_{\mu \nu}^{2} \partial^{\mu} \phi \partial^{\nu} \phi & =-\operatorname{tr}\left(M^{2}\right) \dot{\phi}^{2} \\
& =-\frac{1}{4} \operatorname{tr}\left(M^{2}\right)\left(\dot{\Phi}+\frac{1}{2} \operatorname{tr} L\right)^{2} \\
& =-\frac{1}{4} \dot{\Phi}^{2} \operatorname{tr}\left(M^{2}\right)-\frac{1}{4} \dot{\Phi} \operatorname{tr}\left(M^{2}\right) \operatorname{tr}(L)-\frac{1}{16} \operatorname{tr}\left(M^{2}\right)(\operatorname{tr} L)^{2} \\
& \simeq-\frac{1}{16} \operatorname{tr}\left(L^{2}-M^{2}\right) \operatorname{tr}\left(M^{2}\right)+\frac{1}{4} \operatorname{tr}\left(M^{2} L\right) \operatorname{tr}(L)+\frac{1}{8}\left(\operatorname{tr} M^{2}\right)^{2}-\frac{1}{16} \operatorname{tr}\left(M^{2}\right)(\operatorname{tr} L)^{2} \\
& \simeq-\frac{1}{16} \operatorname{tr}\left(M^{2}\right) \operatorname{tr}\left(L^{2}\right)+\frac{3}{16}\left(\operatorname{tr} M^{2}\right)^{2}+\frac{1}{4} \operatorname{tr}\left(M^{2} L\right) \operatorname{tr}(L)-\frac{1}{16} \operatorname{tr}\left(M^{2}\right)(\operatorname{tr} L)^{2} .
\end{aligned}
$$

The next structure, reduced to one-dimension, is proportional to the previous one

$$
H^{2}(\partial \phi)^{2}=-3 \operatorname{tr}\left(M^{2}\right) \dot{\phi}^{2}=3 H_{\mu \nu}^{2} \partial^{\mu} \phi \partial^{\nu} \phi .
$$

Therefore,

$$
\gamma_{6} H_{\mu \nu}^{2} \partial^{\mu} \phi \partial^{\nu} \phi+\gamma_{7} H^{2}(\partial \phi)^{2}=\left(\gamma_{6}+3 \gamma_{7}\right) H_{\mu \nu}^{2} \partial^{\mu} \phi \partial^{\nu} \phi \equiv \tilde{\gamma}_{6} H_{\mu \nu}^{2} \partial^{\mu} \phi \partial^{\nu} \phi,
$$

and we have put here the new constant $\tilde{\gamma}_{6}$. The condition of duality invariance in one dimension cannot completely determine the action. The last term takes a bit of effort, giving

$$
\begin{aligned}
(\partial \phi)^{4}=\dot{\phi}^{4}= & \frac{1}{16}\left(\dot{\Phi}+\frac{1}{2} \operatorname{tr} L\right)^{4} \\
= & \frac{1}{16} \dot{\Phi}^{4}+\frac{1}{8} \dot{\Phi}^{3} \operatorname{tr} L+\frac{3}{32} \dot{\Phi}^{2}(\operatorname{tr} L)^{2}+\frac{1}{32} \dot{\Phi}(\operatorname{tr} L)^{3}+\frac{1}{(16)^{2}}(\operatorname{tr} L)^{4} \\
& \simeq \frac{1}{16}\left(\operatorname{tr}\left(L^{2}-M^{2}\right)\right)^{2}-\frac{1}{64} \operatorname{tr}\left(L^{2}-M^{2}\right) \operatorname{tr}\left(M^{2}\right)+\frac{3}{128} \operatorname{tr}\left(L^{2}-M^{2}\right)(\operatorname{tr} L)^{2} \\
& -\frac{3}{64} \operatorname{tr}\left(M^{2}\right)(\operatorname{tr} L)^{2}+\frac{1}{(16)^{2}}(\operatorname{tr} L)^{4} .
\end{aligned}
$$

We note here the presence of a term $(\operatorname{tr} L)^{4}$. No other contribution to the left hand side of (3.8) contains such term, nor is it contained on the right-hand side, as shown in (3.9). This means that $(\partial \phi)^{4}$ is incompatible with duality and we can immediately set

$$
\gamma_{8}=0
$$

The complete evaluation of the left hand side of (3.8) thus gives

$$
\begin{aligned}
\mathrm{lhs} \simeq & \gamma_{1}\left(\operatorname{tr}\left(\frac{1}{8} L^{4}-M L M L-\frac{3}{2} M^{2} L^{2}\right)+\frac{3}{8}\left(\operatorname{tr} L^{2}\right)^{2}-\frac{1}{4} \operatorname{tr} M^{2} \operatorname{tr} L^{2}\right) \\
& +\gamma_{2}\left(\operatorname{tr}\left(-\frac{1}{2} M L M L+M^{4}\right)\right)+\gamma_{3}\left(3 \operatorname{tr}\left(M^{4}\right)\right)
\end{aligned}
$$




$$
\begin{aligned}
& +\gamma_{4}\left(4 \operatorname{tr}\left(M^{4}\right)+\left(\operatorname{tr}\left(M^{2}\right)\right)^{2}\right)+\gamma_{5}\left(9\left(\operatorname{tr} M^{2}\right)^{2}\right) \\
& +\tilde{\gamma}_{6}\left(-\frac{1}{16} \operatorname{tr}\left(M^{2}\right) \operatorname{tr}\left(L^{2}\right)+\frac{3}{16}\left(\operatorname{tr} M^{2}\right)^{2}+\frac{1}{4} \operatorname{tr}\left(M^{2} L\right) \operatorname{tr}(L)-\frac{1}{16} \operatorname{tr}\left(M^{2}\right)(\operatorname{tr} L)^{2}\right) .
\end{aligned}
$$

Equating the coefficients of the independent structures on the left- and right-hand sides of (3.8) we get ten equations:

$$
\begin{aligned}
\frac{1}{8} \gamma_{1} & =2 a_{1} \\
-\left(\gamma_{1}+\frac{1}{2} \gamma_{2}\right) & =4 a_{1}+\frac{2}{3}\left(b_{1}+\tilde{b}_{2}\right) \\
-\frac{3}{2} \gamma_{1} & =-8 a_{1}+\frac{2}{3}\left(b_{1}+\tilde{b}_{2}\right), \\
\gamma_{2}+3 \gamma_{3}+4 \gamma_{4} & =2 a_{1}+\frac{2}{3}\left(b_{1}+\tilde{b}_{2}\right), \\
\frac{3}{8} \gamma_{1} & =4 a_{2}-\frac{1}{3} b_{1}+\frac{1}{12} b_{4}, \\
-\frac{1}{4} \gamma_{1}-\frac{1}{16} \tilde{\gamma}_{6} & =-8 a_{2}+\frac{1}{3} b_{1}-\frac{1}{3} \tilde{b}_{2}-\frac{1}{6} b_{4}, \\
\gamma_{4}+9 \gamma_{5}+\frac{3}{16} \tilde{\gamma}_{6} & =4 a_{2}+\frac{1}{3} \tilde{b}_{2}+\frac{2}{3} b_{3}+\frac{1}{12} b_{4}, \\
\frac{1}{4} \tilde{\gamma}_{6} & =\frac{4}{3} b_{3}, \\
-\frac{1}{16} \tilde{\gamma}_{6} & =\frac{1}{3} b_{3}, \\
0 & =-\frac{1}{3} b_{3} .
\end{aligned}
$$

The first four equations and the last three equations are completely equivalent to the following values for the parameters

$$
a_{1}=\frac{1}{16} \gamma_{1}, \quad b_{1}+\tilde{b}_{2}=-\frac{3}{2} \gamma_{1}, \quad b_{3}=0,
$$

as well as the following constraints on the action coefficients:

$$
\gamma_{2}=-\frac{1}{2} \gamma_{1}, \quad 3 \gamma_{3}+4 \gamma_{4}=-\frac{3}{8} \gamma_{1}, \quad \tilde{\gamma}_{6}=0 .
$$

With this information, the remaining equations are

$$
\begin{aligned}
\frac{3}{8} \gamma_{1} & =4 a_{2}-\frac{1}{3} b_{1}+\frac{1}{12} b_{4}, \\
-\frac{1}{4} \gamma_{1} & =-8 a_{2}+\frac{1}{3} b_{1}-\frac{1}{3} \tilde{b}_{2}-\frac{1}{6} b_{4}, \\
\gamma_{4}+9 \gamma_{5} & =4 a_{2}+\frac{1}{3} \tilde{b}_{2}+\frac{1}{12} b_{4} .
\end{aligned}
$$


Interestingly, if we add the three of them, the right-hand side vanishes and we get one more constraint for the action coefficients:

$$
\gamma_{4}+9 \gamma_{5}=-\frac{1}{8} \gamma_{1}
$$

There are no more constraints from these equations and the full list of constraints is then

$$
\gamma_{2}=-\frac{1}{2} \gamma_{1}, \quad 3 \gamma_{3}+4 \gamma_{4}=-\frac{3}{8} \gamma_{1}, \quad \gamma_{4}+9 \gamma_{5}=-\frac{1}{8} \gamma_{1}, \quad \tilde{\gamma}_{6}=\gamma_{8}=0 .
$$

We can parameterize the coefficients $\gamma_{3}, \gamma_{4}$, and $\gamma_{5}$ in terms of a parameter $t$, and $\gamma_{6}$ and $\gamma_{7}$ in terms of a parameter $u$ (recalling that $\tilde{\gamma}_{6}=\gamma_{6}+3 \gamma_{7}=0$ ). We then get

$$
\begin{aligned}
\mathcal{L}(\gamma)= & \gamma_{1}\left(R_{\mu \nu \rho \sigma}^{2}-\frac{1}{2} H H R+\left(\frac{1}{24}+t\right) H^{4}+\left(-\frac{1}{8}-\frac{3}{4} t\right)\left(H_{\mu \nu}^{2}\right)^{2}\right. \\
& \left.+\frac{1}{12} t\left(H^{2}\right)^{2}+u H_{\mu \nu}^{2} \partial^{\mu} \phi \partial^{\nu} \phi-\frac{1}{3} u H^{2}(\partial \phi)^{2}\right)
\end{aligned}
$$

If we take $t=u=0$ we recover the known T-duality invariant action. Up to an overall constant, the eight coefficients in the action are really seven coefficients. From these seven we have determined five, since we have two free parameters. The $u$ parameter dependence is such that reduced to one dimension it disappears. One can quickly check that the $t$ dependence also vanishes in a reduction to one dimension. The constraint of T-duality in the reduction thus had no hope to determine $t$ nor $u$.

\section{Green-Schwarz term and T-duality at $\mathcal{O}\left(\alpha^{2}\right)$}

We now turn to a different application. The goal it to investigate what the double field theory (DFT) constructed in [13] is in terms of conventional field variables. This theory, which we call $\mathrm{DFT}^{-}$[15], features the deformed gauge transformations of the Green-Schwarz mechanism [22], as shown in [14]. In conventional language this implies that the field strength $H$ must be replaced by the improved field strength $\widehat{H}$ that includes the ChernSimons term built from the Christoffel connection. Thus, the minimal action consistent with the two-derivative theory and gauge invariance reads

$$
S=\int d^{D} x \sqrt{-g} e^{-2 \phi}\left(R+4(\partial \phi)^{2}-\frac{1}{12} \widehat{H}^{2}\right)
$$

where

$$
\widehat{H}_{\mu \nu \rho}(b, \Gamma)=3\left(\partial_{[\mu} b_{\nu \rho]}+\alpha^{\prime} \Omega(\Gamma)_{\mu \nu \rho}\right),
$$

with the Chern-Simons three-form

$$
\Omega(\Gamma)_{\mu \nu \rho}=\Gamma_{[\mu|\beta|}^{\alpha} \partial_{\nu} \Gamma_{\rho] \alpha}^{\beta}+\frac{2}{3} \Gamma_{[\mu|\beta|}^{\alpha} \Gamma_{\nu|\gamma|}^{\beta} \Gamma_{\rho] \alpha}^{\gamma}
$$

The full $\mathrm{DFT}^{-}$action might contain order $\alpha^{2}$ terms beyond those following from the Chern-Simons modification in the above minimal action, but this is not required by gauge 
invariance: the minimal action is exactly gauge-invariant under the deformed gauge transformations of the Green-Schwarz mechanism. The purpose of this section is to test duality invariance in order to decide if the above minimal action could be the complete action of $\mathrm{DFT}^{-}$. We will see that while this minimal action satisfies duality invariance to order $\alpha^{\prime}$, it does not satisfy duality invariance to order $\alpha^{\prime 2}$. Thus the above action is not duality complete, and we conclude that $\mathrm{DFT}^{-}$contains further higher-derivative invariants, whose determination we leave for future work.

In order to reduce the action to one dimension one first verifies that the only nonvanishing Chern-Simons components are

$$
3 \Omega(\Gamma)_{0 i j}=-\frac{1}{2} g^{k l} \dot{g}_{k[i} \ddot{g}_{j] l} .
$$

It then follows that the only non-vanishing component of $\widehat{H}$ is

$$
\widehat{H}_{0 i j}=\dot{b}_{i j}-\frac{1}{2} \alpha^{\prime} g^{k l} \dot{g}_{k[i} \ddot{g}_{j] l},
$$

and in matrix notation:

$$
\widehat{H}_{0}{ }^{i}{ }_{j}=\left(M-\frac{1}{4} \alpha^{\prime}[L, \dot{L}]\right)^{i}{ }_{j} .
$$

In order to simplify the evaluation of traces we often use the transposition properties

$$
L^{T}=g L g^{-1}, \quad M^{T}=-g M g^{-1}, \quad \dot{L}^{T}=g \dot{L} g^{-1} .
$$

Together with $\operatorname{tr} Q=\operatorname{tr} Q^{T}$, these allow us to show that

$$
\operatorname{tr}\left(M^{2 k+1} L^{p}\right)=0 \quad \text { for any integers } k, p \geq 0 .
$$

A short computation then gives for the $\widehat{H}^{2}$ term in the Lagrangian

$$
-\frac{1}{12} \widehat{H}_{\mu \nu \rho}^{2}=-\frac{1}{4} \operatorname{tr}(\widehat{H} \widehat{H})=-\frac{1}{4} \operatorname{tr} M^{2}-\frac{1}{4} \alpha^{\prime} \operatorname{tr}(M \dot{L} L)+\frac{1}{32} \alpha^{\prime 2} \operatorname{tr}\left(L^{2} \dot{L}^{2}-(L \dot{L})^{2}\right) .
$$

The $\alpha^{\prime}$ correction here is actually removable by a field redefinition, thus making it clear that to $\mathcal{O}\left(\alpha^{\prime}\right)$ the test of T-duality invariance works out. Indeed, by the replacement $\dot{L} \rightarrow M^{2}+\dot{\Phi} L$ we have

$$
\operatorname{tr}(M \dot{L} L) \simeq \operatorname{tr}\left(M^{3} L\right)+\dot{\Phi} \operatorname{tr}\left(M L^{2}\right)=0,
$$

since these traces are zero. Even though the $\alpha^{\prime}$ correction is removable, for the following analysis we will keep this term, but it is convenient to rewrite it in terms of a metric field equation. To do this we first note that the metric and $b$-field variation in the two-derivative reduced theory (2.59) gives

$$
\delta\left(-\frac{1}{8} e^{-\Phi} \operatorname{tr}\left[(\eta \dot{\mathcal{H}})^{2}\right]\right)=\frac{1}{2} e^{-\Phi} \operatorname{tr}\left[\delta b g^{-1} \mathcal{B} g^{-1}-\delta g g^{-1} \mathcal{G} g^{-1}\right],
$$

where $\mathcal{G}$ and $\mathcal{B}$ are given by

$$
\begin{aligned}
g^{-1} \mathcal{G} & \equiv \dot{L}-M^{2}-\dot{\Phi} L, \\
g^{-1} \mathcal{B} & \equiv \dot{M}-M L-\dot{\Phi} M .
\end{aligned}
$$


Using the identities (4.8), we thus have

$$
\operatorname{tr}(M \dot{L} L)=\operatorname{tr}\left(L M\left(g^{-1} \mathcal{G}+M^{2}+\dot{\Phi} L\right)\right)=\operatorname{tr}\left(L M g^{-1} \mathcal{G}\right) .
$$

It is convenient to symmetrize the factor multiplying $\mathcal{G}$, which we do by adding the trace of the transposed matrix and dividing by two:

$$
\operatorname{tr}(M \dot{L} L)=\frac{1}{2} \operatorname{tr}\left([L, M] g^{-1} \mathcal{G}\right) .
$$

Thus, the reduced action finally takes the form

$$
S_{\text {red }}=\int d t e^{-\Phi}\left(-\dot{\Phi}^{2}-\frac{1}{8} \operatorname{tr}(\eta \dot{\mathcal{H}})^{2}-\frac{1}{8} \alpha^{\prime} \operatorname{tr}\left([L, M] g^{-1} \mathcal{G}\right)+\frac{1}{32} \alpha^{\prime 2} \operatorname{tr}\left(L^{2} \dot{L}^{2}-(L \dot{L})^{2}\right)\right) .
$$

We are now ready to test the duality invariance of the above action. To this end we consider the most general duality covariant action to order $\left(\alpha^{\prime}\right)^{2}$,

$$
S_{\text {dual }}=\int d t e^{-\Phi}\left(-\dot{\Phi}^{2}-\frac{1}{8} \operatorname{tr}(\eta \dot{\mathcal{H}})^{2}+\alpha^{\prime 2} \mathcal{L}^{(2)}\right)
$$

where, given the general classification in (2.78), we have

$$
\mathcal{L}^{(2)}=a_{1} \operatorname{tr}(\eta \dot{\mathcal{H}})^{6}+a_{2} \operatorname{tr}(\eta \dot{\mathcal{H}})^{4} \operatorname{tr}(\eta \dot{\mathcal{H}})^{2}+a_{3}\left(\operatorname{tr}(\eta \dot{\mathcal{H}})^{2}\right)^{3} .
$$

We have to allow for field redefinitions in $S_{\text {dual }}$, implemented by the following replacements inside the generalized metric $\mathcal{H}(g, b)$ :

$$
\begin{aligned}
& g \rightarrow g+\alpha^{\prime} \delta^{(1)} g+\alpha^{2} \delta^{(2)} g, \quad \Delta \equiv g^{-1} \delta^{(1)} g, \\
& b \rightarrow b+\alpha^{\prime} \cdot 0+\alpha^{\prime 2} \delta^{(2)} b .
\end{aligned}
$$

The goal is to choose these redefinitions in such a way that we obtain the dimensionally reduced action (4.15). Note that to first order in $\alpha^{\prime}$ we only redefine the metric, but to second order in $\alpha^{\prime}$ both the metric and the antisymmetric tensor are redefined. In order to compute the effect of the $\delta^{(2)}$ redefinitions to order $\alpha^{\prime 2}$ we just need the first variation of the two-derivative term $\operatorname{tr}(\eta \dot{\mathcal{H}})^{2}$ given in (4.11). To compute the effect of the $\delta^{(1)} g$ redefinition to order $\alpha^{\prime 2}$ we need the second variation of the two-derivative term under a change of the metric. Denoting this change of the metric by $\Delta$,

$$
g \rightarrow g+\delta g, \quad \Delta \equiv g^{-1} \delta g,
$$

a calculation gives

$$
\begin{aligned}
-\left.\frac{1}{8} e^{-\Phi} \operatorname{tr}(\eta \dot{\mathcal{H}})^{2}\right|_{g+\delta g}=-\left.\frac{1}{8} e^{-\Phi} \operatorname{tr}(\eta \dot{\mathcal{H}})^{2}\right|_{g} & -\frac{1}{4} e^{-\Phi} \operatorname{tr}\left(-2 \Delta M^{2}-2 L \dot{\Delta}\right) \\
& -\frac{1}{4} e^{-\Phi} \operatorname{tr}\left(\Delta M \Delta M+2 \Delta^{2} M^{2}-\dot{\Delta} \dot{\Delta}+2 \dot{\Delta} \Delta L\right) .
\end{aligned}
$$

The second order variation is on the second line of the right-hand side. 
We now perform the replacement (4.18) in the duality covariant action (4.16). Using the general first variation (4.11), the second variation from (4.20), and letting $\Delta \equiv g^{-1} \delta^{(1)} g$ we find

$$
\begin{aligned}
\left.S_{\text {dual }}\right|_{\text {rep }}= & \int d t e^{-\Phi}\left(-\dot{\Phi}^{2}-\frac{1}{8} \operatorname{tr}(\eta \dot{\mathcal{H}})^{2}-\frac{1}{2} \alpha^{\prime} \operatorname{tr}\left[\Delta g^{-1} \mathcal{G}\right]\right. \\
& +\frac{1}{2} \alpha^{\prime 2} \operatorname{tr}\left[\delta^{(2)} b g^{-1} \mathcal{B} g^{-1}-\delta^{(2)} g g^{-1} \mathcal{G} g^{-1}\right] \\
& \left.-\frac{1}{4} \alpha^{\prime 2} \operatorname{tr}\left(\Delta M \Delta M+2 \Delta^{2} M^{2}-\dot{\Delta} \dot{\Delta}+2 \dot{\Delta} \Delta L\right)+\alpha^{\prime 2} \mathcal{L}^{(2)}+\mathcal{O}\left(\alpha^{\prime 3}\right)\right) .
\end{aligned}
$$

Comparing with the dimensionally reduced action (4.15), we infer that we need to choose

$$
\Delta=\frac{1}{4}[L, M]
$$

in order to match it to first order in $\alpha^{\prime}$. Note that this is not a duality covariant field redefinition. For this choice we find for the second-order variation

$$
\begin{aligned}
&-\frac{1}{4} \operatorname{tr}\left(\Delta M \Delta M+2 \Delta^{2} M^{2}-\dot{\Delta} \dot{\Delta}+2 \dot{\Delta} \Delta L\right) \\
& \simeq \frac{1}{32} \operatorname{tr}\left(M L^{3} M L-M L^{2} M L^{2}+M^{4} L^{2}-M^{3} L M L\right) \\
& \quad+\frac{1}{8} \dot{\Phi}^{2} \operatorname{tr}\left(M L M L-M^{2} L^{2}\right) .
\end{aligned}
$$

Thus, inserting this into (4.21), we get

$$
\begin{aligned}
\left.S_{\text {dual }}\right|_{\text {rep }}= & \int d t e^{-\Phi}\left(-\dot{\Phi}^{2}-\frac{1}{8} \operatorname{tr}(\eta \dot{\mathcal{H}})^{2}-\frac{1}{8} \alpha^{\prime} \operatorname{tr}\left([L, M] g^{-1} \mathcal{G}\right)\right. \\
& +\frac{1}{2} \alpha^{\prime 2} \operatorname{tr}\left[\delta^{(2)} b g^{-1} \mathcal{B} g^{-1}-\delta^{(2)} g g^{-1} \mathcal{G} g^{-1}\right] \\
& +\frac{1}{32} \alpha^{\prime 2} \operatorname{tr}\left(M L^{3} M L-M L^{2} M L^{2}+M^{4} L^{2}-M^{3} L M L\right) \\
& \left.+\frac{1}{8} \alpha^{\prime 2} \dot{\Phi}^{2} \operatorname{tr}\left(M L M L-M^{2} L^{2}\right)+\alpha^{\prime 2} \mathcal{L}^{(2)}+\mathcal{O}\left(\alpha^{\prime 3}\right)\right)
\end{aligned}
$$

We now note that up to further redefinitions we can replace

$$
L^{2} \dot{L}^{2}-(L \dot{L})^{2} \simeq M^{4} L^{2}-M^{2} L M^{2} L
$$

where it is easy to see that no dilaton terms are produced. Therefore we can add to the above Lagrangian the term

$$
\frac{1}{32} \alpha^{\prime 2} \operatorname{tr}\left(L^{2} \dot{L}^{2}-(L \dot{L})^{2}\right)-\frac{1}{32} \alpha^{\prime 2} \operatorname{tr}\left(M^{4} L^{2}-M^{2} L M^{2} L\right)
$$


by absorbing the field redefinitions into the still undetermined $\delta^{(2)} b$ and $\delta^{(2)} g$. This, together with the use of (2.44) for the term on the last line of the Lagrangian, gives

$$
\begin{aligned}
\left.S_{\text {dual }}\right|_{\text {rep }}= & \int d t e^{-\Phi}\left(-\dot{\Phi}^{2}-\frac{1}{8} \operatorname{tr}(\eta \dot{\mathcal{H}})^{2}-\frac{1}{8} \alpha^{\prime} \operatorname{tr}\left([L, M] g^{-1} \mathcal{G}\right)+\frac{1}{32} \alpha^{2} \operatorname{tr}\left(L^{2} \dot{L}^{2}-(L \dot{L})^{2}\right)\right. \\
& +\frac{1}{2} \alpha^{\prime 2} \operatorname{tr}\left[\delta^{(2)} b g^{-1} \mathcal{B} g^{-1}-\delta^{(2)} g g^{-1} \mathcal{G} g^{-1}\right] \\
& +\frac{1}{32} \alpha^{\prime 2} \operatorname{tr}\left(M L^{3} M L-M L^{2} M L^{2}+M^{2} L M^{2} L-M^{3} L M L\right) \\
& \left.+\frac{1}{32} \alpha^{\prime 2} \operatorname{tr}\left(L^{2}-M^{2}\right) \operatorname{tr}\left(M L M L-M^{2} L^{2}\right)+\alpha^{\prime 2} \mathcal{L}^{(2)}+\mathcal{O}\left(\alpha^{\prime 3}\right)\right) .
\end{aligned}
$$

Let us now compare this with the dimensionally reduced action (4.15),

$$
S_{\text {red }}=\int d t e^{-\Phi}\left(-\dot{\Phi}^{2}-\frac{1}{8} \operatorname{tr}(\eta \dot{\mathcal{H}})^{2}-\frac{1}{8} \alpha^{\prime} \operatorname{tr}\left([L, M] g^{-1} \mathcal{G}\right)+\frac{1}{32} \alpha^{2} \operatorname{tr}\left(L^{2} \dot{L}^{2}-(L \dot{L})^{2}\right)\right) \text {. }
$$

This coincides exactly with the first line of (4.27). Therefore, the hypothesis that the action is duality invariant requires that we can choose the duality covariant terms in $\mathcal{L}^{(2)}$ so that the final two lines of (4.27) are zero up to field and lapse redefinitions, thereby determining in particular $\delta^{(2)} g$ and $\delta^{(2)} b$ in the second line. By the procedure explained in section 2 (see eq. (2.45)) this requires that we can choose coefficients $a_{1}, \ldots, a_{3}$ and a function $X$ such that

$$
\begin{aligned}
0 \simeq & \frac{1}{32} \operatorname{tr}\left(M L^{3} M L-M L^{2} M L^{2}+M^{2} L M^{2} L-M^{3} L M L\right) \\
& +\frac{1}{32} \operatorname{tr}\left(L^{2}-M^{2}\right) \operatorname{tr}\left(M L M L-M^{2} L^{2}\right) \\
& +a_{1} \operatorname{tr}(\eta \dot{\mathcal{H}})^{6}+a_{2} \operatorname{tr}(\eta \dot{\mathcal{H}})^{4} \operatorname{tr}(\eta \dot{\mathcal{H}})^{2}+a_{3}\left(\operatorname{tr}(\eta \dot{\mathcal{H}})^{2}\right)^{3} \\
& +\left(\dot{\Phi}^{2}-\frac{1}{4} \operatorname{tr}\left(L^{2}-M^{2}\right)\right) X .
\end{aligned}
$$

We now make the most general ansatz for the function $X$ to this order in derivatives by writing $X=Y+\tilde{X}$, where

$$
Y=\alpha_{1} \operatorname{tr} L^{4}+\alpha_{2} \operatorname{tr}\left(L^{2} M^{2}\right)+\alpha_{3} \operatorname{tr}(M L M L)+\alpha_{4} \operatorname{tr} M^{4},
$$

is the most general ansatz with a single trace, no dilaton derivatives, and an even number of $M$ 's. The term $\tilde{X}$ then includes dilaton derivatives and or multiple traces. This implies that it cannot contribute relevant terms with single traces. ${ }^{3}$

\footnotetext{
${ }^{3}$ Single trace terms in (4.29) can arise from $\tilde{X}$ terms of the form $\dot{\Phi}^{k} W$, where $W$ has a single trace. Inserted in (4.29), the single-trace terms can only arise from $\dot{\Phi}^{k+2} W$. The recursive relation (2.43) then implies that the single-trace contribution arises from $\dot{\Phi}^{2} W^{\prime} \cdots{ }^{\prime}$, where $W$ is primed-differentiated $k$ times. The effect of this contribution amounts to additive changes to the coefficients $\alpha_{i}$ in (4.30), since $Y$ includes all possible single traces. This is an irrelevant contribution (if one only cares about single traces, as we do) since the coefficients in $Y$ are already completely general. Finally, one can quickly check that a $\dot{\Phi}^{4}$ in $\tilde{X}$ can only give multiple traces.
} 
The idea now is to show that the single trace part of (4.29) cannot be satisfied. To extract the single traces from the last line of (4.29) we recall the identity (2.41). Since all terms in $Y$ have $k=4$, the contribution to the single trace from $\dot{\Phi}^{2} Y$ is proportional to $Y^{\prime \prime}$

$$
\left.\dot{\Phi}^{2} Y\right|_{\text {s.t. }} \simeq \frac{2}{k(2 k-1)} Y^{\prime \prime} \simeq \frac{1}{14} Y^{\prime \prime} .
$$

We find

$$
\begin{aligned}
Y^{\prime \prime}=\operatorname{tr}[ & 2\left(2 \alpha_{1}+\alpha_{2}+\alpha_{3}\right) M L M L^{3}+\left(4 \alpha_{1}+\alpha_{2}\right) M^{2} L^{4}+\left(\alpha_{2}+2 \alpha_{3}\right) M L^{2} M L^{2} \\
& +\left(8 \alpha_{1}+5 \alpha_{2}+4 \alpha_{3}+4 \alpha_{4}\right) M^{4} L^{2}+\left(4 \alpha_{1}+3 \alpha_{2}+2 \alpha_{3}+4 \alpha_{4}\right) M^{2} L M^{2} L \\
& \left.+2\left(3 \alpha_{2}+4 \alpha_{3}+4 \alpha_{4}\right) M^{3} L M L+2\left(\alpha_{2}+\alpha_{3}+2 \alpha_{4}\right) M^{6}\right] .
\end{aligned}
$$

Since nowhere here there is an $L^{6}$ we cannot get the full $\operatorname{tr}(\eta \dot{\mathcal{H}})^{6}$ and therefore we must have $a_{1}=0$. Moreover, as $\operatorname{tr}(\eta \dot{\mathcal{H}})^{6}$ is the only duality invariant single trace term to this order in derivatives, this means that we must cancel the full set of four single trace terms on the first line of (4.29). In order to see that no solution exists it suffices to collect terms in $Y^{\prime \prime}$ proportional to $M^{2}$ :

$$
\begin{aligned}
\left.\dot{\Phi} Y\right|_{\text {s.t. }}= & \left(4 \alpha_{1}^{\prime}+2 \alpha_{2}^{\prime}+2 \alpha_{3}^{\prime}\right) \operatorname{tr}\left(M L^{3} M L\right)+\left(\alpha_{2}^{\prime}+2 \alpha_{3}^{\prime}\right) \operatorname{tr}\left(M L^{2} M L^{2}\right) \\
& +\left(4 \alpha_{1}^{\prime}+\alpha_{2}^{\prime}\right) \operatorname{tr}\left(M^{2} L^{4}\right)+\mathcal{O}\left(M^{4}\right),
\end{aligned}
$$

where we defined $\alpha_{i}^{\prime}=\frac{1}{14} \alpha_{i}$. To cancel the single traces in (4.29) we need

$$
4 \alpha_{1}^{\prime}+2 \alpha_{2}^{\prime}+2 \alpha_{3}^{\prime}=-\frac{1}{32}, \quad \alpha_{2}^{\prime}+2 \alpha_{3}^{\prime}=\frac{1}{32}, \quad 4 \alpha_{1}^{\prime}+\alpha_{2}^{\prime}=0 .
$$

The use of the third equation means that the first and second equations become, respectively,

$$
\alpha_{2}^{\prime}+2 \alpha_{3}^{\prime}=-\frac{1}{32}, \quad \alpha_{2}^{\prime}+2 \alpha_{3}^{\prime}=\frac{1}{32},
$$

which has no solution. This proves that duality does not hold to $\mathcal{O}\left(\alpha^{\prime 2}\right)$ for the action in (4.1).

\section{Conclusions}

We have improved on a method by Meissner to test T-duality invariance of actions with $\alpha^{\prime}$ corrections. The method is now systematic enough that it can be used to test duality covariance to $\mathcal{O}\left(\alpha^{\prime 2}\right)$. It works with an arbitrary field basis, so the analysis can begin with the simplest form of the action that can be obtained by covariant field redefinitions. We have emphasized a built-in limitation of the method: there are non-trivial linear combinations of terms that give zero upon dimensional reduction to one dimension. Such linear combinations of terms cannot be constrained by this test.

The above test of T-duality invariance of $\alpha^{\prime}$ corrections does not suffice to prove that a given action is duality invariant to a given order in $\alpha^{\prime}$. It provides a necessary but not sufficient condition for duality invariance. For the bosonic or heterotic string a direct proof 
of T-duality invariance to order $\alpha^{\prime}$ would be furnished by an extension of the MaharanaSchwarz analysis [6] to order $\alpha^{\prime}$. An exactly duality-invariant effective action almost surely would require terms of all orders in $\alpha^{\prime}$. While the test of T-duality can be applied to string theories in the critical dimension, it could also be applied to the low-energy limits and derivative corrections that arise after arbitrary compactifications, as long as there remains spatial dimensions so that continuous T-duality would emerge upon further compactification on tori.

The power of a double field theory formulation is that it proves T-duality just by its existence. The doubled $\alpha^{\prime}$ geometry of [13] furnishes an exactly T-duality invariant action with $\alpha^{\prime}$ corrections. This theory, called $\mathrm{DFT}^{-}$, is a duality invariant completion of the Green-Schwarz mechanism. We have at present little idea how this action looks in terms of conventional field variables beyond first order in $\alpha^{\prime}$. We applied our test to learn that the action in conventional variables must have terms beyond those that arise from the minimal Green-Schwarz modification of the $b$-field field strength in the kinetic terms.

\section{Acknowledgments}

We thank Ashoke Sen and Krzysztof Meissner for useful discussions on tests of T-duality. The work of O.H. is supported by a DFG Heisenberg fellowship. The work of B.Z. is supported by the U.S. Department of Energy (DoE) under the cooperative research agreement DE-FG02-05ER41360.

Open Access. This article is distributed under the terms of the Creative Commons Attribution License (CC-BY 4.0), which permits any use, distribution and reproduction in any medium, provided the original author(s) and source are credited.

\section{References}

[1] A. Giveon, M. Porrati and E. Rabinovici, Target space duality in string theory, Phys. Rept. 244 (1994) 77 [hep-th/9401139] [INSPIRE].

[2] G. Veneziano, Scale factor duality for classical and quantum strings, Phys. Lett. B 265 (1991) 287 [INSPIRE].

[3] K.A. Meissner and G. Veneziano, Symmetries of cosmological superstring vacua, Phys. Lett. B 267 (1991) 33 [INSPIRE].

[4] A. Sen, $O(d) \times O(d)$ symmetry of the space of cosmological solutions in string theory, scale factor duality and two-dimensional black holes, Phys. Lett. B 271 (1991) 295 [INSPIRE].

[5] S.F. Hassan and A. Sen, Twisting classical solutions in heterotic string theory, Nucl. Phys. B 375 (1992) 103 [hep-th/9109038] [InSPIRE].

[6] J. Maharana and J.H. Schwarz, Noncompact symmetries in string theory, Nucl. Phys. B 390 (1993) 3 [hep-th/9207016] [INSPIRE].

[7] O. Hohm, A. Sen and B. Zwiebach, Heterotic Effective Action and Duality Symmetries Revisited, JHEP 02 (2015) 079 [arXiv: 1411.5696] [INSPIRE]. 
[8] W. Siegel, Superspace duality in low-energy superstrings, Phys. Rev. D 48 (1993) 2826 [hep-th/9305073] [INSPIRE].

[9] W. Siegel, Manifest duality in low-energy superstrings, in proceedings of the International Conference on Strings 93, Berkeley, California, U.S.A., May 24-29 1993, ITP-SB-93-50 [hep-th/9308133] [INSPIRE].

[10] C. Hull and B. Zwiebach, Double Field Theory, JHEP 09 (2009) 099 [arXiv:0904.4664] [INSPIRE].

[11] O. Hohm, C. Hull and B. Zwiebach, Background independent action for double field theory, JHEP 07 (2010) 016 [arXiv: 1003.5027] [inSPIRE].

[12] O. Hohm, C. Hull and B. Zwiebach, Generalized metric formulation of double field theory, JHEP 08 (2010) 008 [arXiv: 1006.4823] [INSPIRE].

[13] O. Hohm, W. Siegel and B. Zwiebach, Doubled $\alpha^{\prime}$-geometry, JHEP 02 (2014) 065 [arXiv: 1306.2970] [INSPIRE].

[14] O. Hohm and B. Zwiebach, Green-Schwarz mechanism and $\alpha^{\prime}$-deformed Courant brackets, JHEP 01 (2015) 012 [arXiv: 1407.0708] [INSPIRE].

[15] O. Hohm and B. Zwiebach, Double field theory at order $\alpha^{\prime}$, JHEP 11 (2014) 075 [arXiv: 1407.3803] [INSPIRE].

[16] D. Marqués and C.A. Núñez, T-duality and $\alpha^{\prime}$-corrections, JHEP 10 (2015) 084 [arXiv: 1507.00652] [INSPIRE].

[17] K.A. Meissner, Symmetries of higher order string gravity actions, Phys. Lett. B 392 (1997) 298 [hep-th/9610131] [INSPIRE].

[18] H. Godazgar and M. Godazgar, Duality completion of higher derivative corrections, JHEP 09 (2013) 140 [arXiv:1306.4918] [INSPIRE].

[19] G. Exirifard and M. O'Loughlin, Two and three loop $\alpha^{\prime}$ corrections to T-duality: Kasner and Schwarzschild, JHEP 12 (2004) 023 [hep-th/0408200] [INSPIRE].

[20] R.R. Metsaev and A.A. Tseytlin, Order $\alpha^{\prime}$ (Two Loop) Equivalence of the String Equations of Motion and the $\sigma$-model Weyl Invariance Conditions: Dependence on the Dilaton and the Antisymmetric Tensor, Nucl. Phys. B 293 (1987) 385 [INSPIRE].

[21] O. Hohm and B. Zwiebach, Double metric, generalized metric and $\alpha^{\prime}$-deformed double field theory, Phys. Rev. D 93 (2016) 064035 [arXiv: 1509.02930] [InSPIRE].

[22] M.B. Green and J.H. Schwarz, Anomaly Cancellation in Supersymmetric D = 10 Gauge Theory and Superstring Theory, Phys. Lett. B 149 (1984) 117 [InSPIRE]. 